\title{
Obstruction of the Ureter by Endometriosis: A Case Report
}

\author{
1) Haemmerle B., 2) Shahin O., 1) Keller N., 1) Schmid S., 3) Buerki N.
}

1) Gynaecology and Obstetrics, Grabs, Switzerland. 2) Urology, Merian Iselin Klinik, Basel, Switzerland 3) Gynaecology and Obstetrics, University Hospital, Basel, Switzerland

\section{Problem statement}

The prevalence of endometriosis (6-10\%) is not a rare disease. However, the incidence seems to be higher due to the often long delay until the diagnosis. An affection of the urogenital organs is rare (1-2\%), but nevertheless as differential diagnosis important.

\section{Methods}

A 34-year old woman presented herself with repetitive pain on the right flank to exclude a suspected congenital ureteral stenosis. A previous magnetic resonance imaging showed a hydronephrosis on the right without detection of a cause of ureteral obstruction. The pain occurred only during menstruation.

\section{Results}

The filiform ureteral stenosis diagnosed in the retrograd uretro-cystography was treated by the dilatation and placement of a double-j-catheter. The histological findings of the ureteral biopsy showed an unspecific inflammation without endometriosis. Because of persisting pain extended and cycled diagnostics with ultrasound and magnetic resonance imaging were carried out and showed a nodule of endometriotic implant of $3 \mathrm{~cm}$ in the right broad ligament. The end ometriotic implant spread around the right ureter and led to ureteral obstruction despite double-j-catheter. So a hormonal treatment with a gestagen-only pill was introduced. In the following diagnostic laparasocopy the appendix vermiformis was also suspected of endometriotic implants and therefore removed. In the same surgery the resection of the impaired distal ureter with intravesical re-implantation (PolitanoLeadbetter-Procedure) was successfully performed. In the follow-up care (after 10 days) the micturating cystourethrogram and the voiding cystourethrogram (after three months) showed no vesicoureteral reflux and a normal bladder voiding. The hormonal treatment was carried on.

\section{Conclusion}

If a young women in reproductive age suffers from ureteral obstruction, it is pivotal to think of endometriosis as a differential diagnosis after excluding the most frequent causes such as kidney stones or tumours. The prognosis of extrinsic ureteral obstruction by endometriosis is much better than an intrinsic endometriosis of the ureter. Overall it is crucial to continue the hormonal treatment.

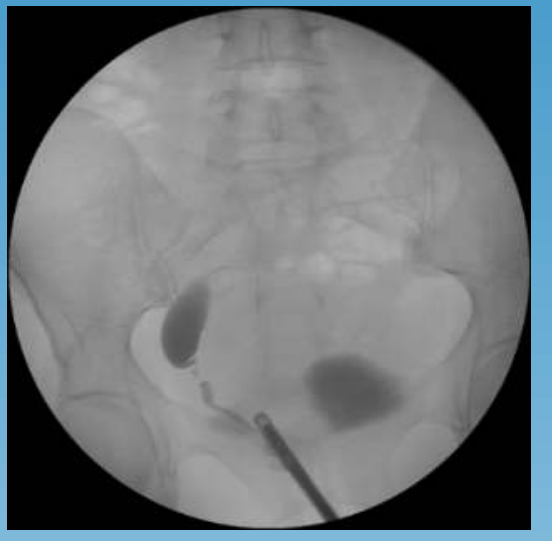

Fig. 1: Retrograd uretro-cystography

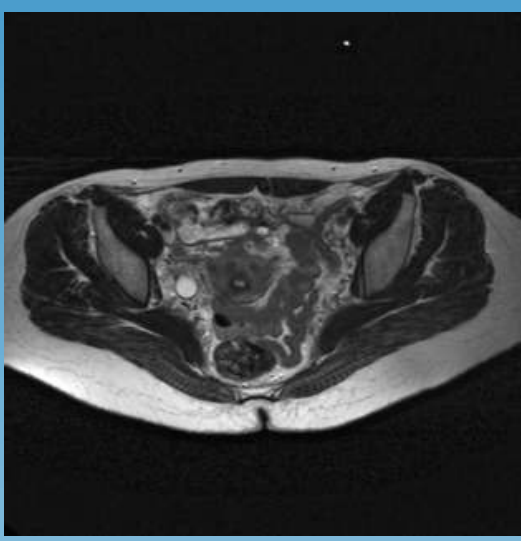

Fig. 2: Endometriotic implant showed in the magnetic resonance imaging

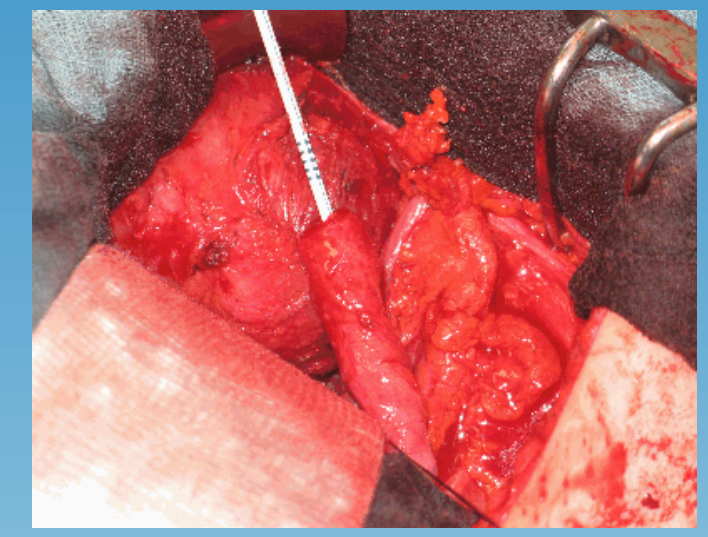

Fig. 3: Resection of the impared distal ureter

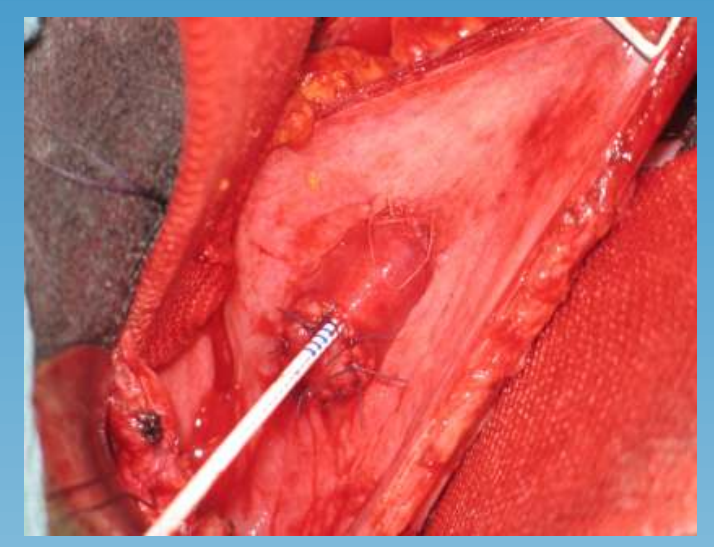

Fig. 4: Intravesical reimplantation of the ureter (Politano-LeadbetterProcedure)

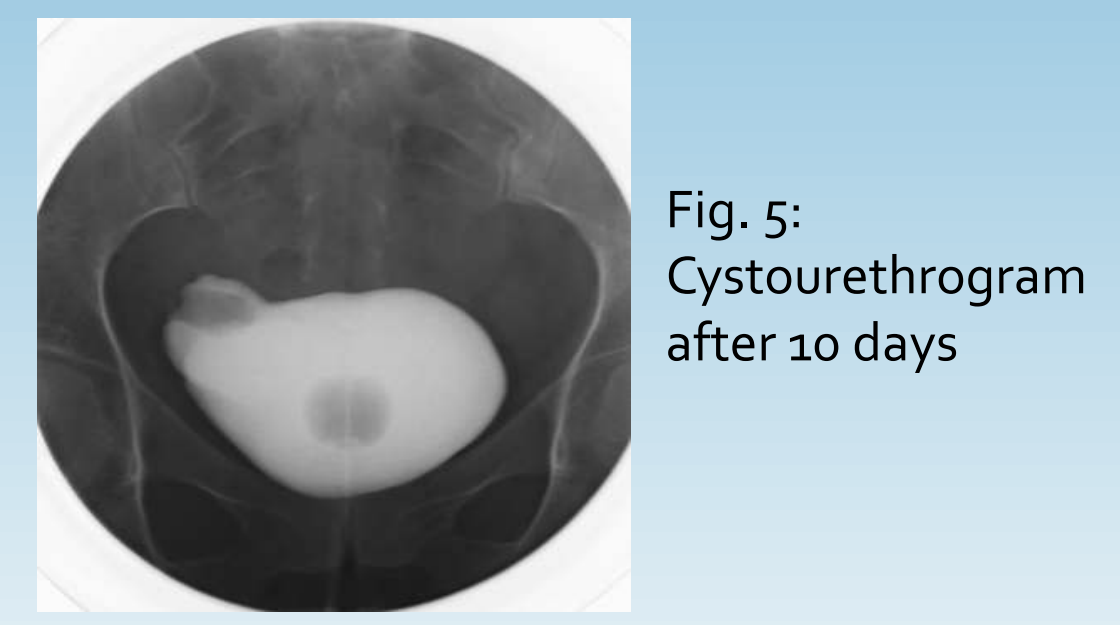

Fig. 6: Voiding cystourethrogram after three months 